УДК 62.52

05.00.00 Технические науки

О ВОЗМОЖНОСТЯХ РЕАЛИЗАЦИИ
ПОДСИСТЕМ УПРАВЛЕНИЯ SMАRТНОUSE

Мунтян Евгения Ростиславна

Старший преподаватель кафедры

вычислительной техники

Email: ermuntyan@sfedu.ru

Скачко Олег Павлович

Студент, Email: chaegolik@gmail.com

Южный федеральный университет, Таганрог,

Россия

В статье описано назначение систем SmartHouse, отмечены их достоинства и недостатки. Выявлены устойчивые тенденции внедрения таких систем в жизнь современных людей, обоснована актуальность их разработки.

Приведено подробное описание системы умного дома, представлена обобщенная структура системы SmartHouse. Выполнен обзор существующих подходов к реализации систем управления SmartHouse. В качестве примера рассмотрена подсистема управления освещением. Разработана структурная схема такой подсистемы. Обоснован выбор технических устройств программно-аппаратной подсистемы управления освещением, приведено их описание. В качестве устройств подсистемы использованы модуль Bluetooth HC-05/06, четырехканальный релейный модуль для подключения к Arduino, плата Arduino Mega 2560. Кратко описан процесс программирования платы Arduino Mega.

Обоснована необходимость удаленного доступа к подсистемам SmartHouse. Рассмотрены существующие стандарты и технологии для решения подобного круга задач. Предложена реализация удаленного доступа к устройствам подсистемы через мобильные системы связи с использованием стандарта Bluetooth. Описано разработанное мобильное приложение, позволяющее управлять устройствами на примере подсистемы освещения. Выполнена апробация модели подсистемы управления освещением, протестировано разработанное программное обеспечение. Внесены предложения по развитию данной работы, предлагается дальнейшее развитие подсистем SmartHouse и реализация удаленного доступа к устройствам системы через Internet

Ключевые слова: SMARTHOUSE, УМНЫЙ ДОМ, УДАЛЕННЫЙ ДОСТУП, ВLUЕТООТН
UDC 62.52

Technical sciences

\section{ABOUT THE APPROACH TO REALIZATION \\ OF MANAGEMENT SUBSYSTEM OF SMARTHOUSE}

Muntyan Evgenia Rostislavna

Department of computer engineering, senior lecturer Email: ermuntyan@sfedu.ru

Skachko Oleg Pavlovich

Student, Email: chaegolik@gmail.com

Southern Federal University, Taganrog, Russia

This article describes the purpose of SmartHouse systems, noting their advantages and disadvantages. We have identified sustainable trends in the implementation of such systems in the life of modern people, and there has been a substantiation of the need for their development. The article provides a detailed description of a SmartHouse system and the generalized structure of the system. Existing approaches to management systems implementation SmartHouse was consider. The subsystem of lighting control was considered as an example. The structural diagram of this subsystem was developed. We have justified the choice of technical devices, hardware and software subsystem of lighting control; we have also given their description. As a subsystem device we use Bluetooth module HC-05/06, four channel relay module to connect to Arduino, Arduino Mega 2560 plate. We briefly describe the process of programming the Arduino Mega plate. The necessity of remote access subsystems was substantiated. We have reviewed existing standards and technologies for solving such problems and proposed the implementation of remote access subsystem via a mobile communication system using the Bluetooth standard. The developed mobile application that allows you to control devices on the example of the lighting subsystem was described. We have performed testing of the model of a subsystem of lighting control and the developed software. The proposal for the development of this work was given. The further development of the subsystems of the SmartHouse and realizing of remote access system through the Internet were offered

Keywords: SMARTHOUSE, REMOTE ACCESS, BLUETOOTH

Doi: 10.21515/1990-4665-124-042 


\section{Введение}

В последнее время отмечается тенденция к улучшению качества жизни, к повышению комфортности жилья. Современное ITориентированное общество готово к применению электронных систем, направленных на продуктивное и эффективное использование рабочего пространства. В качестве технологии разработки подобных систем может использоваться SmartHouse или «Умный дом» [1]. С помощью SmartHouse возможна систематизация инженерии квартиры, дома или же дачного поселка, a планомерное управление домом сможет обеспечить безопасность, экономию и комфорт жилья. Повышение комфорта достигается за счет возможности управления отдельными подсистемами «Умного дома», слаженного взаимодействия всех подсистем между собой и их гармоничного расположения в интерьере дома.

В $[2,3]$ проведен обзорный анализ систем SmartHouse. К достоинствам систем такого типа можно отнести:

- экономию средств за счет снижения расходов на отопление, электроэнергию, водоснабжение и кондиционирование воздуха;

- безопасность благодаря применению датчиков реагирования на утечки, возгорания, поломки оборудования, проникновения в жилище и т.д.;

- комфортные условия проживания.

В тоже время необходимо отметить и недостатки подобных систем:

- дополнительные расходы на оборудование, его установку и обслуживание;

- необходимость приобретения резервного источника бесперебойного питания.

Устойчивые тенденции разработки и внедрения SmartHouse в нашу жизнь заметны, если проанализировать деятельность зарубежных и Российских компаний соответствующей направленности [4-7]. 


\section{Система SmartHouse}

SmartHouse является интегрированной системой безопасности и управления. Такая система, как правило, объединяет в себе функции контроля бытовыми приборами и обеспечения безопасности жилого помещения на разных уровнях. Структура SmartHouse (рис. 1) включает системы электропитания, микроклимата, организации связи и охранную систему. В предложенную структуру возможно добавление других подсистем, например, связанных с развлечениями (домашний кинотеатр и т.д.).

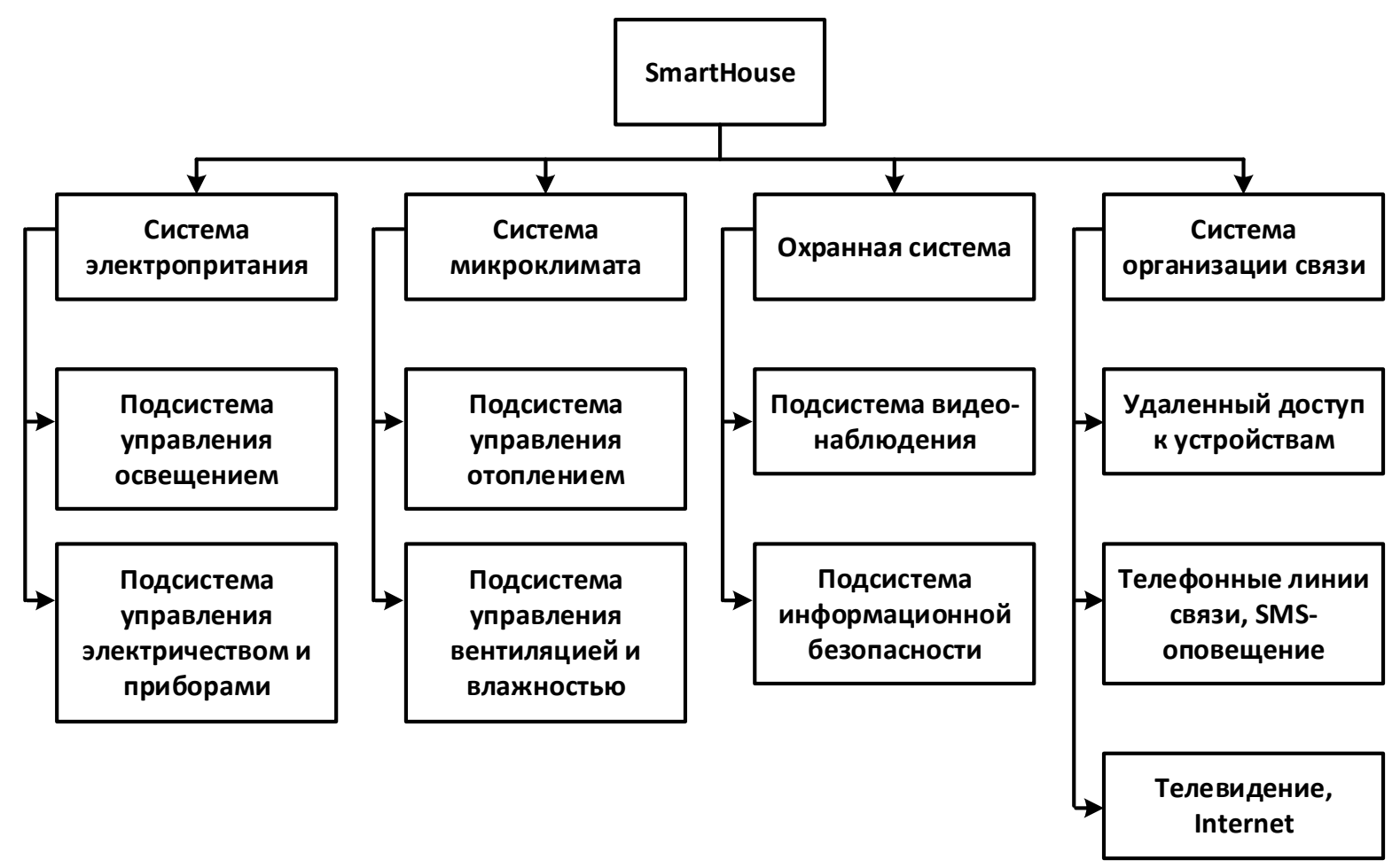

Рисунок 1. Структура SmartHouse

В России активно используются следующие составные части системы «Умного дома»:

- управление освещением и электричеством;

- климат-контроль;

• управления приводами штор и жалюзи;

• д домашний кинотеатр; 
- система мультирум;

- системы безопасности;

- телевидение и интернет;

- связь и телефония.

В данной работе предлагается в качестве примера рассмотреть модель подсистемы управления освещением через мобильное приложение.

\section{Техническая реализация подсистемы управления освещением}

Проанализировав литературные источники [8,9] можно сделать выводы о существующих подходах к реализации систем управления SmartHouse:

- радиоканальные и беспроводные системы.

На российском рынке в нижнем и среднем ценовом сегменте представлены системы, работающие по радиоканалу и передающие управляющие сигналы умного дома по существующим кабельным линиям (X10, Gira radio bus). Несмотря на все технические решения, опыт показывает, что на базе этого оборудования невозможно построить надежную, стабильно работающую систему интеллектуальной, инновационной электрики;

- централизованные проводные системы.

Появление таких систем связано с тем, что увеличение площади умного дома приводит к значительному удорожанию системы, при этом уменьшение площади незначительно влияет на ее стоимость. Идеология централизованных систем хороша при построении развлекательных и мультимедийных систем, но управление светом, электрооборудованием, электрическими системами, лучше реализовывать на децентрализованных или смешанных системах;

- распределенные проводные системы.

На российском рынке в среднем ценовом сегменте отмечается период доминирования систем с так называемым распределенным интеллектом на http://ej.kubagro.ru/2016/10/pdf/42.pdf 
базе оборудования стандарта KNX. Основными недостатками протокола $\mathrm{KNX}$ является большое количество производителей, низкие требования к тестированию совместной работы производимых ими устройств и жесткие требования к монтажу на объекте, что привело к трудностям монтажа посредством электриков под руководством инженеров. Минимальное количество совместимых устройств и весьма ограниченная возможность интеграции не позволяют использовать современные управляющие устройства, делающие управление комфортным и понятным.

В нашем случае целесообразно использовать смешанную структуру системы управления электричеством, которая включает проводное и радиоканальное управление.

На рис. 2 изображена структурная схема, в соответствии с которой разработана модель подсистемы управления освещением для SmartHouse. Опишем назначение и работу основных блоков аппаратной части.

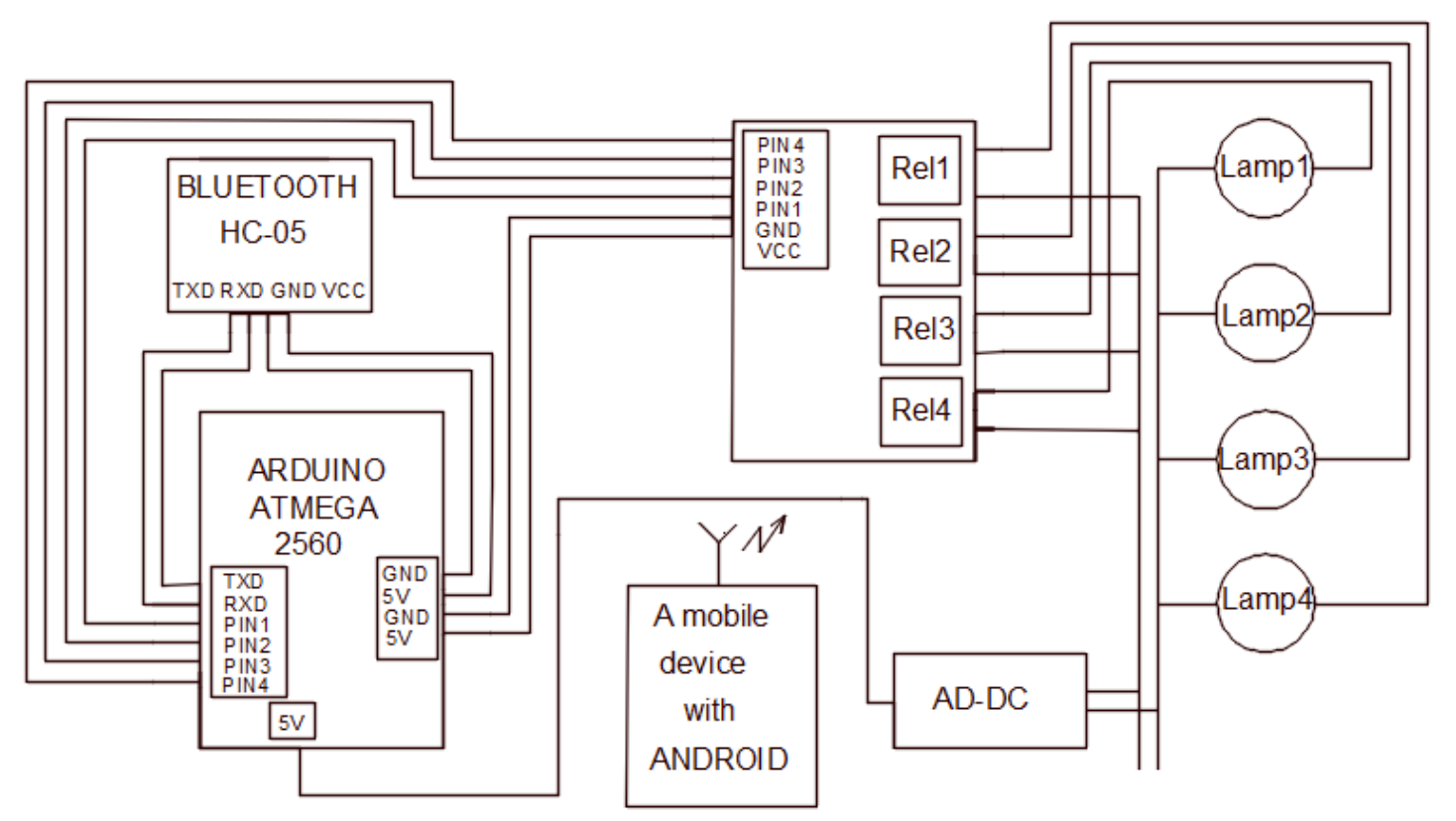

Рисунок 2. Структура подсистемы управления освещением для SmartHouse

Технические устройства, использованные для реализации подсистемы управления освещением: 
- Bluetooth HC-05 соединение с телефоном и компьютером

Модули НС-05/06 представляют собой Bluetooth устройства с дополнительной обвязкой [3]. Выбор именно этого модуля объясняется максимальным удобством и простотой в использовании. Диапазон приемапередачи сигнала до 10 м, что позволяет управлять домом общей площадь до $40 \mathrm{~m}^{2}$;

- Четырехканальный релейный модуль, подключение к Arduino

Управление реле осуществляется следующими элементами: резистором (R1), p-n-p транзистором (VT1), диодом (VD1) и, непосредственно самими реле (Rel1 - Rel4). Также необходимо установление 2 светодиодов для индикации: LED1 (красный) - подачи питания на модуль, LED2 (зеленый) - замыкания реле.

\section{- Плата Arduino Mega 2560}

В качестве управляющего устройства используется микроконтроллер ATmega2560. У платы Arduino Mega 2560 имеется 54 цифровых порта ввода/вывода (GPIO), что необходимо для поддержки нормальной работы микроконтроллера и управления подсистемой освещения.

Платы Arduino Mega могут быть запрограммированы с помощью специального программного обеспечения: UART-загрузчика и утилиты AVRDUDE, которые используются программной средой Arduino IDE для загрузки программы пользователя в память микроконтроллера. Микроконтроллер ATmega2560, установленный на плате, поставляется с программатором, который позволяет загрузить программу пользователя без внешнего программирования контроллера. В программной среде Arduino IDE написан скетч, который впоследствии загружается в микроконтроллер через USB-кабель. 


\section{Организация удаленного доступа к подсистеме}

Для реализации сетевого сопряжения умного дома возможно использование различных технологий: Bluetooth, ZigBee (или 802.15.4/Zigbee), Wi-Fi и WiMAX [10,11]. Каждая из этих сетей ориентирована на решение определенного круга задач.

WiMAX - телекоммуникационная технология, разработанная с целью предоставления универсальной беспроводной связи на больших расстояниях (десятки км) для широкого спектра устройств (от рабочих станций и портативных компьютеров до мобильных телефонов).

При помощи Wi-Fi, например, можно: связываться на средних расстояниях (до 100м) с относительно большими скоростями передачи данных; получать доступ беспроводных устройств в корпоративные сети и Интернет при передаче видео и аудио.

Стандарт Bluetooth в свою очередь передает данные на малых расстояниях (до 10м) и с меньшей скоростью.

В случае использования ZigBee сеть передает небольшие объемы данных на средние расстояния (до 100м), но главная особенность необходимость минимального энергопотребления приемо-передающего устройства. При помощи данной сети можно реализовать сложные схемы мониторинга и управления практически в любой сфере.

Создавая «умный дом», необходимо найти устойчивую, надежную, и бюджетную систему связи между всеми устройствами, являющимися составляющими SmartHouse. Самым рациональным вариантом является использование беспроводной связи, а именно технологии ZigBee, что связано с возможностью построения на основе нее различных видов управления. Критериями выбора могут также служить: низкая энергопотребляемость, отсутствие необходимости широкого канала и экономичность. 
Сейчас для организации доступа к различным устройствам актуально использование мобильных приложений. В [12] описаны подходы к решению данного вопроса, приведен алгоритм доступа к подсистеме SmartHouse c использованием мобильного приложения. На настоящее время в качестве удаленного доступа к реализованной подсистеме использован стандарт передачи данных Bluetooth. Разработано и протестировано мобильное приложение для управления подсистемой SmartHouse.

\section{Заключение}

Несмотря на то, что в настоящее время огромное количество компаний предлагают свои услуги по реализации систем SmartHouse, актуальны собственные разработки в этой области. Это обусловлено доступностью информации о существующих подходах к разработкам и комплектующих устройств, что позволяет осуществлять выбор оптимальных решений.

Система «умный дом» представляет из себя комплекс элементов, позволяющих реализовать полноценную автоматизацию здания. Такая система прежде всего предполагает автоматизацию бытовой деятельности.

В статье описана реализация программно-аппаратной модели SmartHouse на примере подсистемы управления освещением, рассмотрены вопросы организации удаленного доступа к подсистеме по радиоканалу Bluetooth c использованием мобильного приложения. Выполнена апробация модели подсистемы управления освещением, протестировано разработанное программное обеспечение. В качестве продолжения работ в данной области предлагается реализация необходимых подсистем управления и обеспечение возможности удаленного доступа к инженерным системам через Internet. 


\section{Литература}

1. Кадырова Л.Ш. «Умный дом»: идеология или технология. Л.Ш. Кадырова // Международный научно-исследовательский журнал. - 2013. - № 5 - С. 86-87. (http://research-journal.org/arch/umnyj-dom-ideologiya-ili-texnologiya/).

2. Скачко О.П. SmartHouse: плюсы и минусы. О.П. Скачко, Ю.С. Звягинцева, E.P. Мунтян Сборник статей I Всероссийской научно-практической конференции «Информационные системы и технологии: фундаментальные и прикладные исследования». - Таганрог: Изд-во ЮФУ, 2016. - С.166-169.

3. Скачко О.П. Умный дом: поэтапное создание. О.П. Скачко, Ю.С. Звягинцева, Е.Р. Мунтян // Сборник статей I Всероссийской научно-практической конференции «Информационные системы и технологии: фундаментальные и прикладные исследования». - Таганрог: Изд-во ЮФУ, 2016. - С.169-171.

4. Умный дом вчера, сегодня и завтра / Сайт «Инженерные системы для дома». (http://www.220help.ru/textparts.php?tid=605).

5. Монтаж и установка безопасной системы Умный дом / Сайт компании A.P.Technology. - (http://www.aptech.ru/).

6. Умный дом для каждого / Сайт ООО "Смарт Групп". (http://smarthomegroup.ru).

7. Проект умного дома / Сайт «Интеллектуальные системы» (http://www.intellect66.ru/projects/).

8. Смирнова А. Проводные и беспроводные решения на производстве. Что выбрать? А. Смирнова // журнал «Беспроводные технологии». - 2015. - №4 (http://wireless-e.ru/articles/experience/2015_04_56.php).

9. Байчаров С. Выбор технологии беспроводного обмена данными для решения задач автоматизации систем жизнеобеспечения офисно-производственных помещений. С. Байчаров // журнал «Беспроводные технологии». - 2007. - №2 - (http://wirelesse.ru/articles/technologies/2007_2_58.php).

10. Агафонов Н. Технологии беспроводной передачи данных ZigBee, BlueTooth, Wi-Fi. Н. Агафонов // журнал «Беспроводные технологии». - 2006. - №1 (http://www.wireless-e.ru/articles/bluetooth/2006_1_10.php).

11. Колыбельников А.И. Обзор технологий беспроводных сетей. А.И. Колыбельников // Труды МФТИ. - 2012. - т.4, №2. - (https://mipt.ru/upload/6b4/f_g7c9arphcxl1tgs.pdf).

12. Скачко О.П. Организация доступа к подсистеме SmartHouse с использованием мобильного приложения. О.П. Скачко, Е.А. Алексеева, Е.Р. Мунтян // Сборник статей II Всероссийской научно-технической конференции «Фундаментальные и прикладные аспекты компьютерных технологий и информационной безопасности». - 2016. - С. 87 89.

\section{References}

1. Kadyrova L.Sh. «Umnyy dom»: ideologiya ili tekhnologiya. L.Sh. Kadyrova // Mezhdunarodnyy nauchno-issledovatelskiy zhurnal. - 2013. - № 5 - S. 86-87. (http://researchjournal.org/arch/umnyj-dom-ideologiya-ili-texnologiya/).

2. Skachko O.P. SmartHouse: plyusy i minusy. O.P. Skachko, Yu.S. Zvyagintseva, E.R. Muntyan Sbornik statey I Vserossiyskoy nauchno-prakticheskoy konferentsii «Informatsionnye sistemy i tekhnologii: fundamentalnye i prikladnye issledovaniya». Taganrog: Izd-vo YUFU, 2016. - S.166-169. 
3. Skachko O.P. Umnyy dom: poetapnoe sozdanie. O.P. Skachko, Yu.S. Zvyagintseva, E.R. Muntyan // Sbornik statey I Vserossiyskoy nauchno-prakticheskoy konferentsii «Informatsionnye sistemy i tekhnologii: fundamentalnye i prikladnye issledovaniya». - Taganrog: Izd-vo YUFU, 2016. - S.169-171.

4. Umnyy dom vchera, segodnya i zavtra / Sayt «Inzhenernye sistemy dlya doma». (http://www.220help.ru/textparts.php?tid=605).

5. Montazh i ustanovka bezopasnoy sistemy Umnyy dom / Sayt kompanii A.P.Technology. - (http://www.aptech.ru/).

6. Umnyy dom dlya kazhdogo / Sayt OOO "Smart Grupp". (http://smarthomegroup.ru).

7. Proekt umnogo doma / Sayt «Intellektualnye sistemy» (http://www.intellect66.ru/projects/).

8. Smirnova A. Provodnye i besprovodnye resheniya na proizvodstve. Chto vybrat? A. Smirnova // zhurnal «Besprovodnye tekhnologii». - 2015. - №4 - (http://wirelesse.ru/articles/experience/2015_04_56.php).

9. Baycharov S. Vybor tekhnologii besprovodnogo obmena dannymi dlya resheniya zadach avtomatizatsii sistem zhizneobespecheniya ofisno-proizvodstvennykh pomescheniy. S. Baycharov // zhurnal «Besprovodnye tekhnologii». - 2007. - №2 - (http://www.wirelesse.ru/articles/bluetooth/2006_1_10.php).

10. Agafonov N. Tekhnologii besprovodnoy peredachi dannykh ZigBee, BlueTooth, Wi-Fi. N. Agafonov // zhurnal «Besprovodnye tekhnologii». - 2006. - №1 (http://www.wireless-e.ru/articles/bluetooth/2006_1_10.php).

11. Kolybelnikov A.I. Obzor tekhnologiy besprovodnykh setey. A.I. Kolybelnikov // Trudy MFTI. - 2012. - t.4, №2. - (https://mipt.ru/upload/6b4/f_g7c9-arphcx11tgs.pdf).

12. Skachko O.P. Organizatsiya dostupa k podsisteme SmartHouse s ispolzovaniem mobilnogo prilozheniya. O.P. Skachko, E.A. Alekseeva, E.R. Muntyan // Sbornik statey II Vserossiyskoy nauchno-tekhnicheskoy konferentsii «Fundamentalnye i prikladnye aspekty kompyuternykh tekhnologiy i informatsionnoy bezopasnosti». - 2016. - S. 87-89. 